\title{
Spinless particles in screened Coulomb potential
}

\author{
Nagalakshmi A. Rao ${ }^{1}$, B.A. Kagali* \\ Department of Physics, JnanaBharathi Campus, Bangalore University, Bangalore 560 056, India
}

Received 3 August 2001; received in revised form 2 February 2002; accepted 4 February 2002

Communicated by P.R. Holland

\begin{abstract}
We have obtained the analytic solutions of a relativistic spinless particle in a one-dimensional screened Coulomb potential and illustrate the existence of several genuine bound states. We also address the non-relativistic problem for the same potential and compare the energy spectra in the two cases. Comparisons with the results using the one-dimensional Dirac equation are also made. Numerical computations are done using Mathematica. @ 2002 Elsevier Science B.V. All rights reserved.
\end{abstract}

PACS: 03.65.Ge; 03.65.Pm

Keywords: Screened Coulomb potential; Klein-Gordon equation; Energy eigenvalues; Hypergeometric functions; Mathematica

\section{Introduction}

With the increasing applicability of relativistic wave equations in nuclear physics and other areas, the relativistic bound state solutions using the KleinGordon and Dirac equations play a significant role [1]. Setting apart the computational problem, there are some basic unresolved questions in relativistic theory for treating a general potential. In fact, the way of incorporating a general potential is not unambiguously defined. Thus, it is interesting to study the quantum dynamics of relativistic particles under short-range interactions.

We discuss in this Letter the bound states generated by the one-dimensional Klein-Gordon equation for a

\footnotetext{
* Corresponding author. E-mail address: bakagali@vsnl.net.in (B.A. Kagali).

${ }^{1}$ Maharani's Science College for Women, Bangalore.
}

screened Coulomb potential and work out the energy eigenvalues using Mathematica [2] for a band of depth and range parameters. Comparisons are made with the corresponding non-relativistic values.

\section{The screened Coulomb potential}

The one-dimensional time-independent form of the Klein-Gordon equation for a free particle is

$$
\left[\frac{d^{2}}{d x^{2}}+\frac{E^{2}-m^{2} c^{4}}{c^{2} \hbar^{2}}\right] \Psi(x)=0 .
$$

For a potential $V(x)$, in the vector-coupling scheme, this equation takes the form

$$
\left[\frac{d^{2}}{d x^{2}}+\frac{(E-V(x))^{2}-m^{2} c^{4}}{c^{2} \hbar^{2}}\right] \Psi(x)=0 .
$$


We use the one-dimensional screened Coulomb potential of the type

$V(x)=\frac{-g}{2 a} e^{-|x| / a}$,

where $a$ denotes the screening distance of the potential and $g$, the coupling constant. Close to the origin, the potential has the form

$V(x) \approx \frac{-g}{2 a}+\frac{g}{2 a^{2}}|x|$,

and behaves like a linear potential with a constant background of $(-g / 2 a)$. Far from the origin, this potential decreases exponentially.

Using the natural units, $c=1$ and $\hbar=1$, Eq. (2) reduces to

$\left[\frac{d^{2}}{d x^{2}}-2 E V+V^{2}-q^{2}\right] \Psi=0$,

with $q^{2}=m^{2}-E^{2}$. For bound states $q^{2} \geqslant 0$.

Inserting the screened Coulomb potential in the above equation, we obtain, for $x>0$

$$
\begin{aligned}
& {\left[\frac{d^{2}}{d x^{2}}+2 E\left(\frac{g}{2 a}\right) e^{-x / a}\right.} \\
& \left.+\left(\frac{g}{2 a}\right)^{2} e^{-2 x / a}-q^{2}\right] \Psi=0 .
\end{aligned}
$$

Defining a new variable $y=e^{-x / a}$ Eq. (6) transforms to

$$
\begin{aligned}
& {\left[y^{2} \frac{d^{2}}{d y^{2}}+y \frac{d}{d y}\right.} \\
& \left.+\left(a g E y+\frac{g^{2}}{4} y^{2}-a^{2} q^{2}\right)\right] \Psi(y)=0 .
\end{aligned}
$$

We try a solution of the form $\Psi(y)=y^{r} \Phi\left(y^{s}\right)$. By a proper choice of $r$ and $s$, namely, $r=-1 / 2$ and $s=1$, the first derivative term in $\Phi$ is eliminated and we obtain

$$
\left[\frac{d^{2}}{d y^{2}}+\left\{\frac{g^{2}}{4}+\frac{a g E}{y}+\left(\frac{1 / 4-a^{2} q^{2}}{y^{2}}\right)\right\}\right] \Phi(y)=0 .
$$

Further by putting $z=i y$ and $w=g z$, we transform Eq. (8) to the standard form

$$
\left[\frac{d^{2}}{d w^{2}}+\left\{\frac{-1}{4}+\frac{k}{w}+\left(\frac{1 / 4-\mu^{2}}{w^{2}}\right)\right\}\right] \Phi(y)=0 .
$$

We identify this as the Whittaker differential equation [3] whose solutions vanishing at infinity can be written in terms of the confluent hypergeometric functions,

$$
M_{k \mu}(w)=e^{-w / 2} w^{\mu+1 / 2} M\left(\mu-k+\frac{1}{2}, 2 \mu+1 ; w\right),
$$

where $\mu^{2}=a^{2} q^{2}$ and $k=-i a E$.

In terms of the original variable $x$, we can write the wavefunction for positive $x$ as

$$
\begin{aligned}
\Psi_{>}= & A_{+} \exp \left(-\frac{i g}{2} e^{-x / a}-q x\right)(i g)^{a q+(1 / 2)} \\
& \times M\left(\alpha, \gamma ; i g e^{-x / a}\right),
\end{aligned}
$$

with $\alpha=a q+i a E+0.5$ and $\gamma=2 a q+1$.

Since the potential is symmetric, the wavefunction in the negative $x$ region can be written as

$$
\begin{aligned}
\Psi_{<}= & A_{-} \exp \left(-\frac{i g}{2} e^{x / a}+q x\right)(i g)^{a q+(1 / 2)} \\
& \times M\left(\alpha, \gamma ; i g e^{x / a}\right),
\end{aligned}
$$

with $A_{+}$and $A_{-}$as the normalization constants.

\section{The eigenvalue equation}

The eigenvalue equation is obtained by requiring the wavefunction and its derivative to be continuous at the origin.

For even parity states, matching the wavefunctions at the origin precisely leads to $A_{+}=A_{-}$. Using the identity

$\frac{d M}{d w}=\frac{\alpha}{\gamma} M(\alpha+1, \gamma+1 ; w)$,

and matching the derivatives, we obtain the following eigenvalue equation

$\frac{M(\alpha+1, \gamma+1 ; i g)}{M(\alpha, \gamma ; i g)}=\frac{-(2 a q-i g)}{2(\alpha / \gamma) i g}$.

For odd parity states, we have $\Psi(x)=-\Psi(-x)$. This implies the vanishing of the hypergeometric function at the origin, thus leading to the following eigenvalue 
Table 1

Relativistic ground state energy eigenvalues of the screened Coulomb potential. ( $E$ in units of $m c^{2} a$ in units of $\hbar / m c$ and $g$ in units of $\hbar c$ )

\begin{tabular}{|c|c|c|c|c|c|c|}
\hline \multicolumn{4}{|c|}{ Klein-Gordon } & \multicolumn{3}{|c|}{ Dirac } \\
\hline$g$ & $\begin{array}{c}a=1.0 \\
E\end{array}$ & $\begin{array}{c}a=1.5 \\
E\end{array}$ & $\begin{array}{c}a=2.0 \\
E\end{array}$ & $\begin{array}{c}a=1.0 \\
E\end{array}$ & $\begin{array}{c}a=1.5 \\
E\end{array}$ & $\begin{array}{c}a=2.0 \\
E\end{array}$ \\
\hline 0.5 & 0.939899 & 0.952142 & 0.959686 & 0.9430659 & 0.953582 & 0.960488 \\
\hline 1.0 & 0.830394 & 0.871739 & 0.895305 & 0.842799 & 0.877045 & 0.898154 \\
\hline 1.5 & 0.698923 & 0.778325 & 0.821912 & 0.724936 & 0.789186 & 0.827639 \\
\hline
\end{tabular}

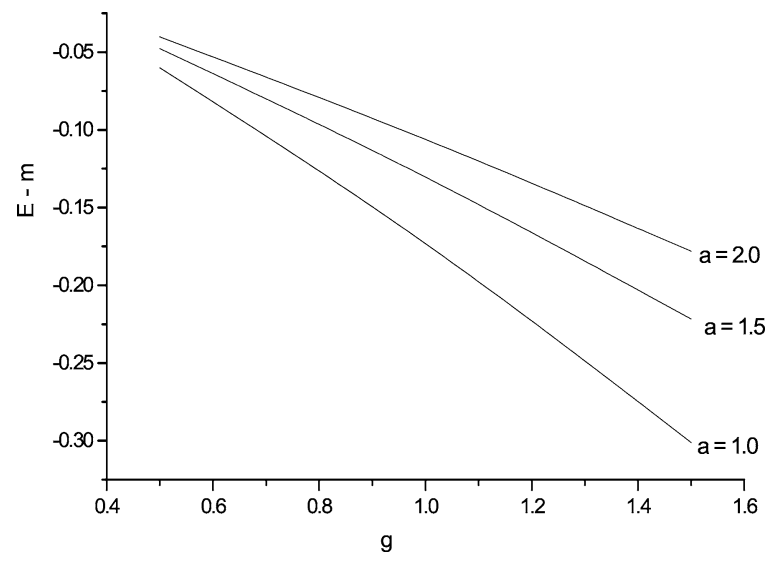

Fig. 1. Ground state energy of a Klein-Gordon particle of mass unity as function of $g$ for various values of $a$.

equation

$M(\alpha, \gamma ; i g)=0$.

These are complicated transcendental equations, the real roots of which can only be found numerically, using computational tools like Mathematica. The energy eigenvalues for the ground state, calculated for typical values of $a$ and $g$ are listed in Table 1. Fig. 1 illustrates the ground state energy level for a particle of mass unity as a function of the coupling constant $g$ for various range parameters. The values obtained by Dominguez-Adame et al. [4] using the Dirac equation are also shown in Table 1, for comparison. For a chosen $a$, besides the ground state, the potential also admits excited states for higher values of $g$. Table 2 shows the binding energies $(e=E-m)$ in units of $m c^{2} . a$ is expressed in units of $\hbar / m c=\lambda$ and $g$ in units of $\hbar c$. Energy profiles for $a=1 \lambda$ as a function of $g$ are shown in Fig. 2.

\subsection{The non-relativistic eigenvalues}

Znojil [5] has pointed out that the Schrödinger equation for a type of screened Coulomb potential serves as a good description of heavy quarks. With a view to comparing the relativistic and the nonrelativistic eigenenergies of the screened Coulomb potential, we solve the one-dimensional Schrödinger equation for the potential in equation (3). The wave equation

$\frac{d^{2} \Psi}{d x^{2}}+\frac{2 m E}{\hbar^{2}} \Psi+\frac{m g}{\hbar^{2} a} e^{-x / a} \Psi=0$,

with the substitution, $z=\beta e^{-x /(2 a)}$, transforms to the well-known Bessel equation,

$z^{2} \frac{d^{2} \Psi}{d z^{2}}+z \frac{d \Psi}{d z}+\left(z^{2}-v^{2}\right) \Psi=0$,

where $\beta^{2}=4 m a g / \hbar^{2}$ and $\nu^{2}=-8 m E a^{2} / \hbar^{2}$.

The admissible solutions in the positive and negative $x$ regions, can respectively, be written in terms of the Bessel functions of first kind as

$\Psi_{>}=C J_{v}\left(\beta e^{-x /(2 a)}\right) \quad$ and $\quad \Psi_{<}=D J_{v}\left(\beta e^{x /(2 a)}\right)$.

Furthermore, imposing the boundary conditions as before, the eigenenergies are obtained from the eigenvalue equations:

$J_{-1+v}(\beta)=J_{1+v}(\beta)$,

for even parity states, and

$J_{v}(\beta)=0$,

for odd parity states. Typical eigenvalues $(E)$ for a 
Table 2

Binding energies of the Klein-Gordon particle of mass unity as a function of the coupling constant $(a=1 \lambda)$

\begin{tabular}{|c|c|c|c|c|c|c|}
\hline$g$ & $e_{0}$ & $e_{1}$ & $e_{2}$ & $e_{3}$ & $e_{4}$ & $e_{5}$ \\
\hline 0.000003 & 0.00000 & & & & & \\
\hline 0.001 & $-4.9863 \times 10^{-7}$ & & & & & \\
\hline 0.01 & $-4.8674 \times 10^{-5}$ & & & & & \\
\hline 0.05 & $-1.1045 \times 10^{-3}$ & & & & & \\
\hline 0.1 & -0.003986 & & & & & \\
\hline 1.0 & -0.169605 & & & & & \\
\hline 1.34739 & -0.259218 & 0.00000 & & & & \\
\hline 2.0 & -0.446557 & -0.019616 & & & & \\
\hline 2.99655 & -0.764824 & -0.107621 & 0.00000 & & & \\
\hline 4.0 & -1.112556 & -0.245776 & -0.026389 & & & \\
\hline 5.0 & -1.480365 & -0.419110 & -0.096912 & & & \\
\hline 5.43505 & -1.646688 & -0.503403 & -0.138937 & 0.00000 & & \\
\hline 6.0 & -1.869575 & -0.619727 & -0.202357 & -0.006835 & & \\
\hline 7.77195 & - & -1.026516 & -0.455835 & -0.102551 & 0.00000 & \\
\hline 9.0 & - & -1.339026 & -0.669744 & -0.220995 & -0.026323 & \\
\hline 10.5222 & - & - & -0.970487 & -0.413258 & -0.119119 & 0.00000 \\
\hline 12.0 & - & - & -1.293405 & -0.638971 & -0.258953 & -0.034261 \\
\hline
\end{tabular}

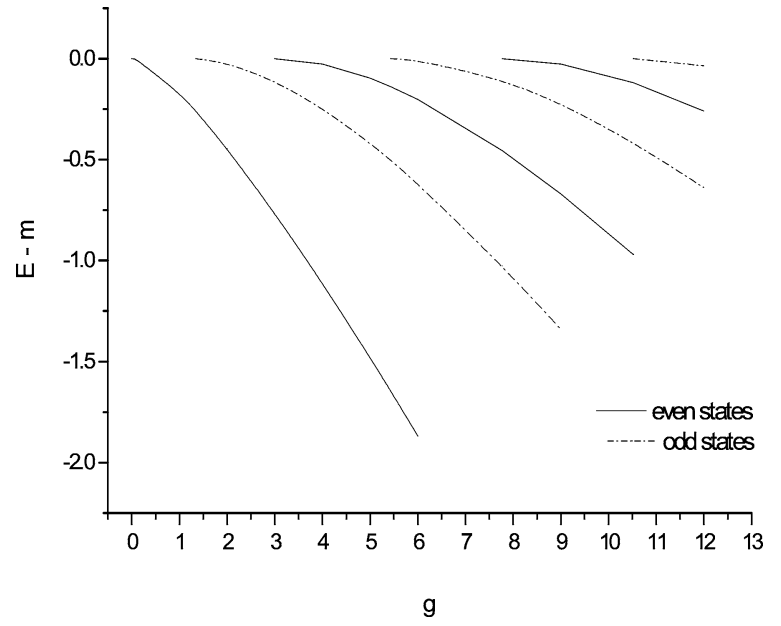

Fig. 2. Energy profile of a Klein-Gordon particle in a screened Coulomb potential $(a=1 \lambda)$ as a function of $g$.

fixed $a$ and different $g$ are listed in Table 3. $E$, $a$ and $g$ are expressed in the same units as before for easy comparison with the relativistic binding energies. Energy fits are illustrated in Fig. 3.

\section{Results and discussion}

While the one-dimensional Coulomb potential presents only scattering states, it is seen that the one- dimensional screened Coulomb potential generates genuine bound states, both relativistically and nonrelativistically. It is evident from Table 1 that our results are consistent with those of Dominguez-Adame et al. and the curves in Fig. 1 show exactly the same trend as those of a Dirac particle. For a chosen $a$, the bound states become deeper and deeper on increasing the coupling constant, and for a fixed $g$, increasing $a$ leads to shallower energies, as expected. Nevertheless, for very small $g$, like $g \cong 0.001$, the relativistic binding energy agrees well with the non-relativistic energy, a feature inherent of relativistic wave equations. For small $g$ only the even parity states occur. As $g$ is increased, we find, at higher energy, an odd parity solution starting at a threshold value. Still higher depth leads to excited states, with the odd and even solutions occurring successively, a feature typical of any bound state problem. It may be noted that for values of $g \leqslant 1$, the relativistic correction is less than $6 \%$, thus defining the potential region as non-relativistic, while for higher values of $g$ the relativistic correction increases steadily and for some value of $g,(E-m)$ reaches -2 , the limiting value admissible for a single particle wave equation.

More importantly, while the energy spectrum admits energies from $+m c^{2}$ to $-m c^{2}$ in the relativistic case, the allowed non-relativistic energies are restricted to those within the well-depth. For a given 
Table 3

Non-relativistic bound state energies of a particle of mass unity as a function of the coupling constant $(a=1 \lambda)$

\begin{tabular}{|c|c|c|c|c|c|c|}
\hline$g$ & $E_{0}$ & $E_{1}$ & $E_{2}$ & $E_{3}$ & $E_{4}$ & $E_{5}$ \\
\hline 0.000003 & 0.00000 & & & & & \\
\hline 0.001 & $-4.9851 \times 10^{-7}$ & & & & & \\
\hline 0.01 & $-4.8557 \times 10^{-5}$ & & & & & \\
\hline 0.05 & $-1.0929 \times 10^{-3}$ & & & & & \\
\hline 0.1 & -0.003913 & & & & & \\
\hline 1.0 & -0.158327 & & & & & \\
\hline 1.44745 & -0.264665 & 0.00000 & & & & \\
\hline 2.0 & -0.408604 & -0.009970 & & & & \\
\hline 3.67324 & -0.894701 & -0.125266 & 0.00000 & & & \\
\hline 4.0 & -0.995583 & -0.158340 & -0.001395 & & & \\
\hline 5.0 & -1.312830 & -0.275158 & -0.021214 & & & \\
\hline 7.62166 & -2.188920 & -0.664770 & -0.160856 & 0.00000 & & \\
\hline 8.0 & -2.319360 & -0.728674 & -0.189413 & -0.000964 & & \\
\hline 12.3096 & -3.854151 & -1.552389 & -0.623772 & -0.125247 & 0.00000 & \\
\hline 15.0 & -4.846379 & -2.134340 & -0.973881 & -0.286827 & -0.027742 & \\
\hline 18.7278 & -6.251758 & -3.002341 & -1.532632 & -0.591003 & -0.146076 & 0.00000 \\
\hline 20.0 & -6.738010 & -3.312050 & -1.739621 & -0.712811 & -0.204710 & -0.004347 \\
\hline
\end{tabular}

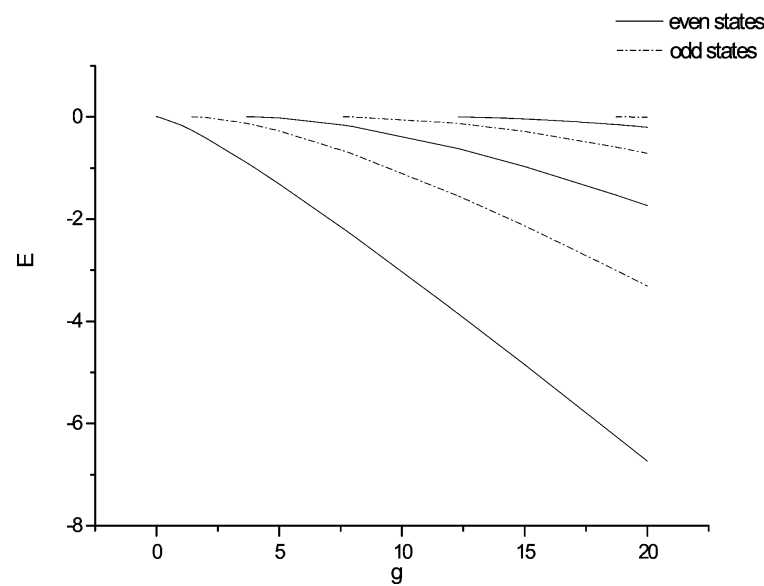

Fig. 3. Energy profile of a non-relativistic particle in a screened Coulomb potential $(a=1 \lambda)$ as a function of $g$.

well-depth, the relativistic particle is more tightly bound as compared to the non-relativistic particle, which may be attributed to the increase in the "effective mass" of the relativistic particle. Thus the energy spectra of our screened Coulomb potential exhibit the general features of relativistic bound states of short range potentials [1].

Our results may find applications in the study of pionic atoms, light quarkonia [6], doped Mott insulators [7], etc.

\section{Acknowledgements}

Thanks are extended to Prof. V. Kamalamma, Principal, Maharani's Science College for Women, Bangalore for encouragement. One of the authors (N.A.R.) is grateful to U.G.C. for the award of fellowship. We would like to thank the referee for drawing our attention to some unforeseen errors and the need for extending our calculations to the non-relativistic and truly relativistic domains.

\section{References}

[1] W. Greiner, Relativistic Quantum Mechanics, Springer, 1995.

[2] S. Wolfram, The Mathematica Book, Wolfram Media, 3rd edn., Cambridge University Press, 1996.

[3] M. Abramowitz, I.A. Stegun, Handbook of Mathematical Functions and Formulas, Graphs and Mathematical Tables, Dover, New York, 1965.

[4] F. Domiguez-Adame, A. Rodrigues, Phys. Lett. A 198 (1995) 275 , and references therein.

[5] M. Znojil, Phys. Lett. A 94 (1983) 120.

[6] M. Znojil, Phys. Lett. A 102 (7) (1984) 289.

[7] B. Mona, J. Sanjeev, Phys. Rev. B 57 (16) (1998) 9521. 